\title{
Rhinologic and Sinonasal Changes in PR3 ANCA Pulmonary VASCULITIS
}

\author{
K. Zycinska, K. A. Wardyn, E. Piotrowska, T.M. Zielonka, H. Zycinski, A. Bogaczewicz, \\ A. Nitsch-Osuch, M. Miskiewicz, R. Smolarczyk \\ Primary Systemic Vasculitis Outpatient Clinic, Department of Family Medicine, Internal and Metabolic Diseases, \\ Warsaw Medical University, Warsaw, Poland
}

\begin{abstract}
Background: Over 70-95\% patients with PR3 ANCA pulmonary vasculitis present with upper respiratory tract symptoms or sings. Nasal cavity usually presents with obstruction and chronic refractory infections (rhinosinusitis) which commonly manifest as bloody discharge or crusting obstruction. Mucopurulent discharge may occur in the acute phase or remission, along with other symptoms suggesting sinusitis. Later on, saddle nose deformities can occur due to collapse of the nasal septum. Other common destruction areas are the maxillary ostia, erosion of the tubinates or damage of soft palate.

Objective: The aim of the study was to characterize pathologies of nasal and sinonasal CT scans in patients with PR3 pulmonary ANCA vasculitis and to establish the CT diagnostic criteria for WG. Between 2005-2009 sinonasal CT visualization was performed in 35 patients (19 female, 16 male) with PR3 ANCA positive WG.

Results: Bony destruction of the nasal cavity was revealed in $15(42.8 \%)$, damage or distortion of the paranasal sinuses in $20(57.1 \%)$, the mastoid cells in 7 $(20 \%)$, and the orbits in $7(20 \%)$ patients. Sclerosing osteitis of the nasal cavity and paranasal sinuses were observed in $11(31.4 \%)$ and in 24 (68.5\%), respectively. Bony thickening of the nasal cavity was shown in 5 $(14.2 \%)$ patients and of the paranasal sinuses in 7 $(20 \%)$ (unilateral in 2 and bilateral in 5 patients). Seven patients $(20 \%)$ had orbital masses; all unilateral. Septal perforation was observed in $11(31.4 \%)$ and saddle nose deformity in $7(20 \%)$ patients.

Conclusions: Maxillary sinuses are regions which are most frequently affected during the course of PR3 ANCA pulmonary vasculitis. CT imagines may be a useful supplement to clinical and activity scoring of WG disease with pulmonary involvement.
\end{abstract}

Key words: rhinosinusitis, paranasal sinus, Wegener's granulomatosis, disease activity

\section{INTRODUCTION}

Wegener's granulomatosis (WG) is a systemic disease characterized by necrotizing granulomatous inflammation of upper and lower airways and glomerulonephri- tis. The pathogenesis of the disease is not well known [1]. Patients with WG frequently have clinical symptoms of respiratory tract infection and ANCA titer elevation. Furthermore, these patients have secondary infection of nasal and paranasal tissues. Over 70-95\% patients with PR3 ANCA pulmonary vasculitis present with upper respiratory tract symptoms or sings. Nasal activity usually presents with nasal obstruction and chronic refractory infections (rhinosinusitis), commonly manifested by bloody discharge, crusting, and local obstruction $[2,3]$. Mucopurulent discharge may occur in the acute phase or remission along with other symptoms suggesting sinusitis. Later on, saddle nose deformities can occur due to collapse of the nasal septum. Other common destruction areas are the maxillary ostia, erosion of the tubinates, or damage of soft palate. Nasal damage may lead to epiphora or inflammation of the tear duct or eye as well as to conductive hearing loss.

The aim of the present study was to determinate the relevance of CT changes as a marker of disease activity and chronicity. To characterize bone abnormalities of the nose, paranasal sinuses, orbits, and mastoid cells in patients with WG, we performed a retrospective study of all patients who underwent CT scanning at our hospital between 2005-2009.

\section{Material And Methods}

The study was approved by a local Ethics Committee. Thirty five patients, 19 female and 16 male subjects, of the mean age $48.8 \pm 11.5(\mathrm{SD})$ (range 16 - 86 years) with biopsy-proven pulmonary Wegener's granulomatosis from the Primary Systemic Vasculitis Outpatients Clinic of the Czerniakowski Hospital in Warsaw, Poland were enrolled into the study. The mean followup of the patients was 42.6 months. All patients fulfilled the American College of Rheumatology criteria for the classification of WG and the Chapel Hill Consensus Conference definition, and also EUVAS ANCA-associated vasculitis definition for WG. Disease activity was confirmed by clinical scoring, laboratory variables, and imaging procedures. DEI and BVAS indexes were determined to measure organ involvement and disease activity. The disease symptoms included chronic rhinosinusitis as defined by the Rhi- 
nosinusitis Task Force criteria [4]. The number and type of sinonasal surgeries for each patient was calculated. The patients were examined with non-enhanced sinonasal CT scans, including axial and coronal views, with bone window and soft tissue window. Slice thickness was 2-3 mm. Enhanced CT scanning and MRI was performed in 7 patients with a pseudotumor of the orbit. Furthermore, allocation and spread of sinonasal filling bone mineralization and infiltration of the soft tissue were retrospectively studied on CT. The following imaging findings were assessed in the area of the nasal cavity: paranasal sinuses, orbits, mastoid cells, thickening, subtotal opacification, air-fluid level, bony destruction, sclerosing osteitis, orbital mass, saddle nose deformity, and septal perforation. A subtotal opacification was diagnosed when the affected sinus was at least opacified in $75 \%$. The CT scans reading was performed by two radiologists. Sclerosing osteitis was determined by an increased density of bone structures on CT imagines. The criteria for a saddle nose deformity were the following: acquired loss of nasal dorsal height and shortening of the nose. Pathologic involvement of the orbital and frontal sinus floor was regarded as an involvement of the orbit and frontal sinus.

\section{REsults}

Sinonasal involvement in WG was noted in $92 \%$ of the patients. Symptoms and signs at the time of the study are presented in Table 1 . Seven $(20 \%)$ patients underwent primary functional endoscopic sinus

Table 1. Overall rhinosinonasal symptoms frequency in Wegener's granulomatosis.

\begin{tabular}{lcc}
\hline Data & $\begin{array}{c}\text { Patients } \\
(\mathrm{n}=35)\end{array}$ & $\begin{array}{c}\text { Percentage } \\
(\%)\end{array}$ \\
\hline Crusting & 29 & 82.8 \\
Nasal obturation & 26 & 74.3 \\
Bloody discharge & 19 & 54.3 \\
Epiphora & 16 & 45.7 \\
Chronic rhinosinusitis & 25 & 71.4 \\
Septal perforation & 11 & 31.4 \\
Saddle-nose deformity & 7 & 20 \\
Mucocele & 8 & 22.6 \\
Nose granulomatous mass & 3 & 8.6 \\
Maxillary mass & 7 & 20 \\
Orbital pseudotumor & 7 & 20 \\
\hline
\end{tabular}

surgery (FESS) or dacryocystorhinotomy (DCR) prior to the study. These included FESS for refractory CRS or mucocele decompression in 5 patients, DCR for epiphora or chronic dacrocistitis in 2 patients, and orbital decompression for pseudotumor of the orbit in one patient. Two $(5.7 \%)$ of the 35 CT scans reviewed were judged to be normal and $33(94.3 \%)$ to have pathologic findings. Subtotal opacifications of the paranasal sinuses were detected in $7(20 \%)$ patients (all bilateral) and of the mastoid cells in $3(8.6 \%)$ patients (all unilateral) (Table 2). These changes were mostly observed in the maxillary sinuses $(42 \%)$, frontal sinuses $(26 \%)$, ethmoidal cells $(20 \%)$, and sphenoidal sinuses $(12 \%)$. Seven $(20 \%)$ of the 35 patients had air-fluid levels in the maxillary sinuses indicating acute sinusitis (unilateral in 4 patients, bilateral in 1 patient). Mucosal thickening of the nasal cavity was observed in 25 $(71.4 \%)$ patients (unilateral in 5 patients and bilateral in 20 patients). Mucosal thickening of the paranasal sinuses was seen in $28(80 \%)$ patients. Bony destruction of the nasal cavity was revealed in $15(42.8 \%)$, the paranasal sinuses in $20(57.1 \%)$, the mastoid cells in 7 $(20 \%)$, and of the orbits in $7(20 \%)$ patients. Sclerosing osteitis of the nasal cavity and paranasal sinuses were observed in $11(31.4 \%)$ and $24(68.5 \%)$ patients, respectively. Bony thickening of the nasal cavity was shown in $5(14.2 \%)$ patients (bilateral) and of the paranasal sinuses in $7(20 \%)$ (unilateral in 2 patients and bilateral in 5 patients). In $7(20 \%)$ patients there were orbital masses (all unilateral). Septal perforation we observed in $11(31.4 \%)$ patients, and saddle nose deformity in $7(20 \%)$ patients.

\section{Discussion}

Sinonasal disease is typical for PR3-ANCA positive vasculitis. Chronic sinonasal involvement may result in rhinosinusitis (CRS), septal perforation, saddle-nose deformity, and paranasal sinus mucocele formation and chronic outflow tract obstruction. According to BVAS-WG index (Modified Birmingham Vasculitis Activity Score for Wegener's granulomatosis) typical otolaryngical features are: retrobulbar mass with proptosis, bloody nasal discharge, nasal crusting, nasal ulcer, chronic rhinosinusitis, new deafness, hoarseness (stridor), mouth ulcer, and swollen salivary gland. All these changes observed in the present study are similar to those in Cannady's et al [5] and Lohrmann's et al [6] studies.

Because all these symptoms are nonspecific, the identification of characteristic radiologic changes may lead to the confirmation of Wegener's granulomatosis

Table 2. Sinonasal CT findings in 35 patients with Wegener's granulomatosis.

\begin{tabular}{lcccc}
\hline \multicolumn{1}{c}{ Location } & Nasal cavity & Paranasal sinuses & Mastoid cells & Orbits \\
\hline Mucosal Thickening & $25(71.5 \%)$ & $28(80 \%)$ & 0 & 0 \\
Subtotal Opacification & $13(37.1 \%)$ & $7(20 \%)$ & $3(8.6 \%)$ & 0 \\
Bony Destruction & $15(42.8 \%)$ & $20(57.1 \%)$ & $7(20 \%)$ & $5(14.2 \%)$ \\
Bony Thickening & $5(14.2 \%)$ & $7(20 \%)$ & $3(8.6 \%)$ & $2(5.7 \%)$ \\
Sclerosing Osteitis & $11(31.4 \%)$ & $24(68.5 \%)$ & $4(11.4 \%)$ & $3(8.6 \%)$ \\
\hline
\end{tabular}


recognitions [7, 8]. Computed tomography scanning is likely to demonstrate a mucosal thickening similar to that typical for chronic sinusitis. The presence of bony erosions of the nasal and sinonasal wall is deeply suspected of a granulomatous disease. Bone erosion always affects the orbital wall (lamina papyracea), nasosinusal wall, and nasal septum, mainly centered with the maxillary sinus unilateral or bilateral. Bone destruction usually involves the nasal septum and extends to the sinonasal wall [9]. Isolated septal perforation should suggest the diagnosis of WG, but differential diagnosis should include traumatic or toxic lesions (chromium salts, cocaine powder). The disease may progress to the palate and soft tissues in the orbital direction. In the present study, bony destruction and bony thickenings were widely observed in the sinonasal regions assessed. Benoudiba et al [10] have reported that the underlying granulomatous process may contribute to the periostitis due to infection, commonly Stapbylococcus aureus in affected sinuses, which may hasten destructive processes. Prognosis of destructive facial lesions depends on many factors: anatomical (skull base, meninges, fossas), microbiological ( $S$. aureus colonization and carries), serological (PR3-ANCA activity).

Bony destruction of the nasal cavity and sinuses observed in CT scans can lead to confirmation of WG diagnosis. CT findings should alert a clinician to perform a PR3-ANCA or c-ANCA test before any attempts on sinonasal surgery. Surgery may be indicated to remove nodular nasal mucosal thickening, or correct punctuate bony destructions, especially located at the septum, bony demineralization, or orbital extension.

Conflicts of interest: No conflicts of interest were declared by the authors in regard to this work.

\section{REFFERENCES}

1. Kallenberg CG. Pathogenesis of PR3-ANCA associated vasculitis. Autoimmun 2008; 30(1-2): 29-36.

2. Pagnoux C, Teixeira L. Wegener's granulomatosis. Presse Med 2007; 36 (5): 860-87.
3. Jayne D. Progress of treatment in ANCA-associated vasculitis. Nephrology 2009; 14(1): 42-8.

4. Benninger MS, Ferguson BJ, Hadley JA, Hamilos DL, Jacobs M, Kennedy DW, Lanza DC, Marple BF, Osguthorpe JD, Stankiewicz JA, Anon J, Denneny J, Emanuel I, Levine H. Adult chronic rhinosinusitis: definitions, diagnosis, epidemiology and pathophysiology. Otolaryngol Head Neck Surg 2003; 129: S1-32.

5. Cannady SB, Batra PS, Koening C, Lorenz RR, Citardi MJ, Langford C, Hoffman GS. Sinonasal Wegener's granulomatosis: a single- institution experience with 120 cases. Laryngoscope 2009; 119; 757-61.

6. Lohrmann C, Uhl M, Warnatz K, Kotter E, Ghanem N, Langer M. Sinonasal computed tomography in patients with Wegener's granulomatosis. J Comput Assist Tomogr 2006; 30: 122-5.

7. Martinez del Pero M, Sivasothy P. Vasculitis of the upper and lower airway. Best Pract Res Clin Rheumatol 2009; 23(3): 403-17.

8. Lloyd G, Lund VJ, Beale T, Howard D. Rhinologic changes in Wegener's granulomatosis. J Laryngol Otol 2002; 116: 565-9.

9. Seo P. Vasculitis: Lessons learned. Curr Opin Rheumatol 2009; 21(1): 1-2.

10. Benoudiba F, Marsot-Dupuch K, Rabia MH, Cabanne J, Bobin S, Lasjaunias P. Sinonasal Wegener's granulomatosis: CT characteristics. Neuroradiology 2003; 45(2): 95-9.

Address for correspondence:

Katarzyna Zycinska

Primary Systemic Vasculitis Outpatient Clinic

Department of Family Medicine,

Internal and Metabolic Diseases

Nephrology Unit

Warsaw Medical University

19/25, Stepinska St.

00-187 Warsaw

Poland

Phone/fax: +4822318 6325

E-mail: kzycinska@poczta.fm 